\title{
Anatomical and functional brain abnormalities in unmedicated major depressive disorder
}

\author{
This article was published in the following Dove Press journal: \\ Neuropsychiatric Disease and Treatment \\ 18 September 2015 \\ Number of times this article has been viewed
}

\author{
Xiao Yang ${ }^{1,2, *}$ \\ Xiaojuan $\mathrm{Ma}^{3, *}$ \\ Mingli $\mathrm{Li}^{1,2}$ \\ Ye Liu' \\ Jian Zhang' \\ Bin Huang ${ }^{4}$ \\ Liansheng Zhao',2 \\ Wei Deng ${ }^{1,2}$ \\ Tao $\mathrm{Li}^{1,2}$ \\ Xiaohong $\mathrm{Ma}^{1,2}$ \\ 'Psychiatric Laboratory and \\ Department of Psychiatry, ${ }^{2} \mathrm{National}$ \\ Key Laboratory of Biotherapy, West \\ China Hospital, Sichuan University, \\ ${ }^{3}$ Chengdu First People's Hospital, \\ Chengdu, ${ }^{4}$ Dong Feng Mao Jian \\ Hospital, Shiyan, People's Republic \\ of China \\ *These authors contributed equally \\ to this work
}

Correspondence: Xiaohong Ma

Psychiatric Laboratory and Department of Psychiatry, West China Hospital, Sichuan University, 37 Guoxue Alley, Wuhou,

Chengdu, Sichuan 61004I, People's

Republic of China

Email maxiaohong@scu.edu.cn
Background: Using magnetic resonance imaging (MRI) and resting-state functional magnetic resonance imaging (rsfMRI) to explore the mechanism of brain structure and function in unmedicated patients with major depressive disorder (MDD).

Patients and methods: Fifty patients with MDD and 50 matched healthy control participants free of psychotropic medication underwent high-resolution structural and rsfMRI scanning. Optimized diffeomorphic anatomical registration through exponentiated lie algebra and the Data Processing Assistant for rsfMRI were used to find potential differences in gray-matter volume (GMV) and regional homogeneity ( $\mathrm{ReHo}$ ) between the two groups. A Pearson correlation model was used to analyze associations of morphometric and functional changes with clinical symptoms.

Results: Compared to healthy controls, patients with MDD showed significant GMV increase in the left posterior cingulate gyrus and GMV decrease in the left lingual gyrus $(P<0.001$, uncorrected). In ReHo analysis, values were significantly increased in the left precuneus and decreased in the left putamen $(P<0.001$, uncorrected $)$ in patients with MDD compared to healthy controls. There was no overlap between anatomical and functional changes. Linear correlation suggested no significant correlation between mean GMV values within regions with anatomical abnormality and ReHo values in regions with functional abnormality in the patient group. These changes were not significantly correlated with symptom severity.

Conclusion: Our study suggests a dissociation pattern of brain regions with anatomical and functional alterations in unmedicated patients with MDD, especially with regard to GMV and ReHo.

Keywords: major depressive disorder, functional magnetic resonance imaging, gray-matter volume, regional homogeneity

\section{Introduction}

By the year 2020, major depressive disorder (MDD) will become the second leading cause of global disease burden. ${ }^{1}$ It is characterized by a persistent depressed mood, alterations in motivation, and pervasive feelings of guilt and worthlessness, and will affect approximately $15 \%$ of the general population. ${ }^{2}$ Although the etiology and pathogenesis of depression is still unclear, the rapid development of neuroimaging technologies have provided improved methods and made it possible to explore brain structure and functional abnormalities in MDD patients. ${ }^{3}$

Past anatomical studies designed to explore whole-brain differences had reported that MDD patients showed smaller gray-matter volume (GMV) in some brain regions, such as the frontal cortex, ${ }^{4}$ temporal gyrus, ${ }^{5}$ putamen, ${ }^{6}$ and caudate. ${ }^{7}$ Meanwhile, GMV was found to be increased in the amygdala, ${ }^{8,9}$ hippocampus, ${ }^{10}$ and other regions ${ }^{11}$ in patients with depression. Some research has found that GMV deficits in the frontal gyrus, temporal lobe, and insula were negatively correlated with depressive symptoms or illness duration in MDD patients. ${ }^{12,13}$ The prefrontal 
cortex volume has been shown to be negatively correlated with risky choices in patients with MDD. ${ }^{14}$ In addition, decreased GMV of the cingulate cortex has been associated with decreased cognitive performance in patients with depression. ${ }^{15}$ These findings imply that GMV abnormalities in patients with depression may be correlated with clinical symptoms. In this study, we used magnetic resonance imaging (MRI) with voxel-based morphometry (VBM), applying the diffeomorphic anatomical registration through exponentiated lie algebra (DARTEL) procedure to investigate the change in GMV in patients with MDD and the relationship between clinical variables.

Resting-state functional MRI (rsfMRI) scans performed on patients who were in a relaxed state of mind with eyes closed has aroused increasing interest since the study of Biswal et al. ${ }^{16}$ Regional homogeneity (ReHo), a frequently used method, is conducted to analyze the similarities of intraregional time series across the whole brain. Moreover, ReHo reflects the temporal synchrony of spontaneous blood oxygen level-dependent signal rather than its density. Therefore, abnormal ReHo may be relevant to changes in temporal aspects of neural activity in regional areas, and can be used to detect abnormal activity in whole-brain regions of psychiatric disorders in the resting state.

As a matter of fact, ReHo has been widely used to explore the pathophysiology of neuropsychiatric disorders, such as Parkinson's disease, ${ }^{17}$ schizophrenia, ${ }^{18}$ attention deficit/ hyperactivity disorder, ${ }^{19}$ autism, ${ }^{20}$ anxiety, ${ }^{21}$ and MDD. ${ }^{22}$ As reported in previous studies, MDD had been associated with abnormal neural activity in some brain regions implicated in emotional regulation, such as the dorsal prefrontal cortex, the amygdala, and other regions. ${ }^{8,23}$ A meta-analysis of neuroimaging studies focused on ReHo of patients with depression found that the medial prefrontal cortex was increased in depression. ${ }^{24}$ In addition, ReHo alterations in depression patients have been identified in many other brain regions, including the anterior cingulate cortex ${ }^{25}$ or precuneus, and have been shown to correlate with symptom severity or disease duration. ${ }^{26}$ A previous study showed that besides patients with MDD, those at high risk for MDD also exhibited significantly decreased ReHo in the right insula and in the left cerebellum. ${ }^{22}$ Furthermore, Wang et al found that patients with MDD displayed a ReHo decrease in the right precuneus after treatment with escitalopram. ${ }^{27}$

These findings showed that functional alterations in brain regions can be identified using ReHo in patients with MDD. Moreover, ReHo changes in the precuneus have been found in response to pharmacological treatment in MDD. In this study, we detected regional neural activity in medication-free patients with MDD in the resting state by using the ReHo method.

Multimodal neuroimaging techniques, such as structural MRI and rsfMRI, were used to explore the pathophysiology of depression. Nevertheless, it is unclear whether these anatomical alterations and functional deficits contribute independently to depression. Previous studies have shown that anatomical and functional brain abnormalities are dissociated in schizophrenia and might contribute independently to the pathophysiology of schizophrenia, ${ }^{28-31}$ while one study reported decreased association between functional activity and regional GMV in schizophrenia. ${ }^{32}$ Recently, a study reported that the dissociation of anatomical and functional abnormalities was also observed in patients with MDD. ${ }^{33}$ To our knowledge, this is the first study that investigated anatomical and functional alterations simultaneously in the same depression patients. The authors investigated abnormalities of GMV and amplitude of low-frequency fluctuation (LFF) in patients with MDD, and found that brain structural and functional deficits contribute independently to depression.

The aim of the current study was to explore the association between brain functional and anatomical deficits in unmedicated patients with MDD. Based on the aforementioned studies, ${ }^{28-31,33}$ we hypothesized that functional and anatomical abnormalities in brain regions would be observed in different brain areas in medication-free patients with MDD. To test this hypothesis, we investigated abnormalities of GMV and ReHo in unmedicated patients with depression, the interrelationship between these alterations, and their relationship with clinical variables.

\section{Patients and methods}

\section{Participants}

Depressive patients included in our study generally visited their psychiatrist because of depressive relapse after quitting medication. At that time, their physician either asked him/ her to contact us or asked his/her permission to be referred to us. Fifty unmedicated patients with MDD were recruited from the Psychiatry Department of West China Hospital of Sichuan University. Major depression was diagnosed by two qualified psychiatrists (XM and ML) using the Structured Clinical Interview according to the Diagnostic and Statistical Manual of Mental Disorders (DSM)-IV criteria. ${ }^{34}$ All patients were assessed and scanned as soon as possible (usually within 3 days) to prevent treatment delay. Patients who had taken psychotropic medications in the previous 3 months before scanning were excluded. In addition, exclusion criteria included age younger than 18 years or older than 60 years, 
history of loss of consciousness, mental retardation, cardiovascular disease, schizophrenia, bipolar disorder, anxiety disorder, neurological illness, and alcohol or drug abuse. Fifty demographically similar (age-, sex-, and education-matched) healthy controls were recruited by poster advertisements from the local area. Healthy controls were interviewed using the Structured Clinical Interview for DSM-IV, non-patient edition, ${ }^{35}$ to assure that none of them had a current or past history of depression or other major physical or neurological illness, or substance abuse.

All participants signed an informed consent form prior to participation in the study. This study was approved by the Ethics Committee of Sichuan University, and was conducted according to the Helsinki Declaration.

\section{HAM-D and HAM-A questionnaires}

All patients were scored by two qualified psychiatrists (XHM and MLL) according to the 17-item Hamilton Depression Rating Scale (HAM-D) and 14-item Hamilton Anxiety Scale (HAM-A) to assess the severity of symptoms. Patients with scores $\geq 18$ on the HAM-D were included in our study.

\section{MRI data acquisition}

All scanning was performed on a 3.0 T MR scanner (Achieva; Philips, Amsterdam, the Netherlands) using an eight-channel phased-array head coil. Foam padding and earplugs were used to minimize head movement and scanner noise. During scanning, participants were often reminded to remain motionless with eyes closed, without falling asleep, and without thinking of anything special (confirmed by subjects immediately after the experiment).

High-resolution T1 images were acquired by 3-D magnetization-prepared rapid gradient-echo sequence as follows: repetition time $8.37 \mathrm{~ms}$, echo time $3.88 \mathrm{~ms}$, flip angle $7^{\circ}$, in-plane matrix resolution $256 \times 256$, field of view $24 \times 24 \mathrm{~cm}^{2}$, and number of slices 188 . A total of 240 volumes of echo-planar images were obtained axially with a gradientecho echo-planar imaging sequence with the following parameters: repetition time $2,000 \mathrm{~ms}$, echo time $3.711 \mathrm{~ms}$, flip angle $7^{\circ}$, in-plane matrix resolution $256 \times 256$, field of view $256 \times 256 \mathrm{~mm}^{2}$, and number of slices 38 . None of the participants had more than $2 \mathrm{~mm}$ maximum displacement in $x, y$, or $z$ and $2^{\circ}$ of angular motion during the whole MRI scan. For each participant, the fMRI scanning lasted for 8 minutes and 6 seconds, and 240 volumes were obtained.

\section{Image processing and analysis}

All structural data were processed using the DARTEL ${ }^{36}$ toolbox with the Statistical Parametric Mapping software package (SPM8; http://www.fil.ion.ucl.ac.uk/spm). VBM preprocessing involved five steps, and followed the standard approach of Ashburner. ${ }^{36}$ The modulated gray-matter images were smoothed with an isotropic Gaussian kernel of $6 \mathrm{~mm}$ full width at half maximum to be used in statistical analysis.

ReHo analysis was performed with the Data Processing Assistant for Resting-State $\mathrm{AMRI}^{37}$ in MatLab (MathWorks, Natick, MA, USA). Individual ReHo maps were generated by calculating Kendall's coefficient of concordance (KCC) of the time series of a given voxel with those of its nearest neighbors (26 voxels) in a voxel-wise analysis. ${ }^{38}$ Zang et al described the formula for calculating the $\mathrm{KCC}$ value in their study. ${ }^{39}$ After calculating the ReHo map voxel by voxel, the resulting fMRI data were then spatially smoothed with a Gaussian kernel of $6 \times 6 \times 6 \mathrm{~mm}^{3}$ full width at half maximum. While functional connectivity approaches measure the temporal correlation of low-frequency fluctuations (LFFs) between remote brain regions, ReHo measures the local synchronization of spontaneous fMRI, ${ }^{40}$ which is based on the assumption that LFFs within a functional cluster will synchronize with neighboring voxels. ${ }^{24}$

\section{Statistical analysis}

Based on SPSS version 17.0, the $\chi^{2}$ test for categorical data and Student's $t$-test for continuous variables were used to evaluate differences in demographic characteristics between patients and controls. Two sample $t$-tests contained in SPM8 were used to test the differences in GMV and ReHo values between patients and controls. Confounding factors were regressed out, including age, sex, education years, and total volume of gray matter and white matter of each subject. Anatomical analyses yielded statistical parametric maps based on a voxel-level height threshold of $P<0.001$ (uncorrected for multiple comparisons). The statistical results of $\mathrm{ReHo}$ were corrected using the AlphaSim program, which is based on Monte Carlo simulations (http://afni.nimh.nih.gov/pub/dist/ doc/manual/AlphaSim.pdf). The statistical threshold for this analysis was set at $P<0.001$.

To determine the overlap between GMV and ReHo results, brain regions with abnormal GMV or ReHo were overlaid on the same template. Furthermore, brain regions with abnormal GMV and ReHo were identified as regions of interest. Mean values of GMV and ReHo were extracted for further Pearson's correlation analysis between these abnormal values and the HAM-D or HAM-A scores in the patient group. Confounding factors were regressed out, including age and sex. 


\section{Results}

\section{Clinical characteristics}

Age (years; MDD 31.12 \pm 9.495 , healthy controls 31.30 \pm 9.307 ), sex ratio (male:female 19:31 in both groups), and years of education (MDD 13.42 \pm 3.387 , healthy controls $13.52 \pm 3.418$ ) were not significantly different between MDD patients and healthy controls $(P>0.05)$ (Table 1). Mean HAM-D and HAM-A scores were $23.10 \pm 4.196$ and $16.12 \pm 5.612$, respectively.

\section{GMV and ReHo alterations in MDD patients}

Relative to healthy controls, patients with MDD showed significantly increased GMV in the left posterior cingulate gyrus (PCG; Montreal Neurological Institute [MNI]: $x=-3$, $y=-48, z=8$, voxels $=208 ; P<0.001$, uncorrected) and significantly decreased GMV in the left lingual gyrus (LG; MNI: $x=-25, y=-64, z=-4$, voxels $=251 ; P<0.001$, uncorrected) (Figure 1). With regard to the ReHo comparison, patients showed significantly increased ReHo values in the left precuneus (MNI: $x=-12, y=-63, z=60$, voxels $=44 ; P<0.001$, uncorrected) and decreased ReHo values in the left putamen (MNI: $x=-27, y=-6, z=-3$, voxels $=28 ; P<0.001$, uncorrected) (Figure 2, Table 2).

\section{Association between morphometric and functional results}

We overlaid the regions with abnormal GMV or ReHo values on the same template, but found no overlap of brain regions. Linear correlation suggested no significant correlation between mean GMV values within regions with anatomical abnormalities and ReHo values in regions with functional abnormality in the patient group.

\section{Correlations between clinical variables and functional/anatomical alterations}

The structural and functional brain alterations in MDD did not significantly correlate with symptom severity in the patient group (Table 3).

\section{Discussion}

Compared to healthy controls, patients with MDD showed significantly increased GMV in the left PCG and decreased GMV in the left LG. Functional analysis showed that patients had increased ReHo values in the left precuneus and decreased ReHo values in the left putamen. No overlap of brain regions with structure or functional alterations was observed in patients. Moreover, no significant correlation between mean GMV values or ReHo values of the regions and clinical variables was found in the depressed group.

In our study, VBM-DARTEL identified increased GMV in the left PCG and decreased GMV in the left LG. Previous studies may have found gray-matter reduction in the CG, identifying these abnormalities in elderly depressed patients, ${ }^{41}$ depressed adolescents, ${ }^{42}$ and patients with psychotic depression ${ }^{43}$ while these structural brain abnormalities were particularly found in patients with a longer course of illness. In addition, depression in late life is frequently associated with medical comorbidity. ${ }^{44}$ Other studies may have been limited by relatively small sample size ${ }^{45}$ effect of medication, or the current mood state. ${ }^{46}$

Meanwhile, other investigators also found that selected samples of MDD patients free of medical comorbidity showed a decrease in CG volume when compared with controls. ${ }^{47}$ However, a meta-analysis showed increased gray matter in the cingulate cortex in medication-washout patients. ${ }^{48}$ The GMV of cingulate regions in these studies varied. The main factors of such inconsistent results may include mixed samples of patients with MDD. A previous study reported that the PCG was innervated by the serotonergic system ${ }^{49}$ which is associated with vulnerability or pathophysiology of depression. ${ }^{50}$ Some studies suggested it may play an important role in the integration of emotional behaviors ${ }^{51}$ and in the interactions between emotion and cognition. ${ }^{52}$ Vogt et al reported that the PCG may partially underlie self-referential emotional processing, ${ }^{53,54}$ and it has been further identified to be preferentially involved in affective evaluation of incoming stimuli, crucial to the initiation of aggression..$^{55}$

Table I Characteristics of MDD patients and HCs

\begin{tabular}{llll}
\hline Characteristics & MDD & HCs & $P$-value \\
\hline $\mathrm{n}$ (males/females) & $50(19 / 3 \mid)$ & $50(19 / 31)$ & $1.000^{\mathrm{a}}$ \\
Age (years), mean \pm SD & $31.12 \pm 9.495$ & $31.30 \pm 9.307$ & $0.924^{\mathrm{b}}$ \\
Education (years completed), mean \pm SD & $13.42 \pm 3.387$ & $13.52 \pm 3.418$ & $0.883^{\mathrm{b}}$ \\
Disease duration (months), mean \pm SD & $9.84 \pm 12.59$ & - & - \\
Number of episodes, mean \pm SD & $1.30 \pm 0.58$ & - & - \\
HAM-D score, mean \pm SD & $23.10 \pm 4.196$ & - & - \\
HAM-A score, mean \pm SD & $16.12 \pm 5.612$ & - & - \\
\hline
\end{tabular}

Notes: a Obtained by $\chi^{2}$ test; bobtained by $t$-test.

Abbreviations: MDD, major depressive disorder; HCs, healthy controls; SD, standard deviation; HAM-D, Hamilton Depression Rating Scale; HAM-A, Hamilton Anxiety Rating Scale. 

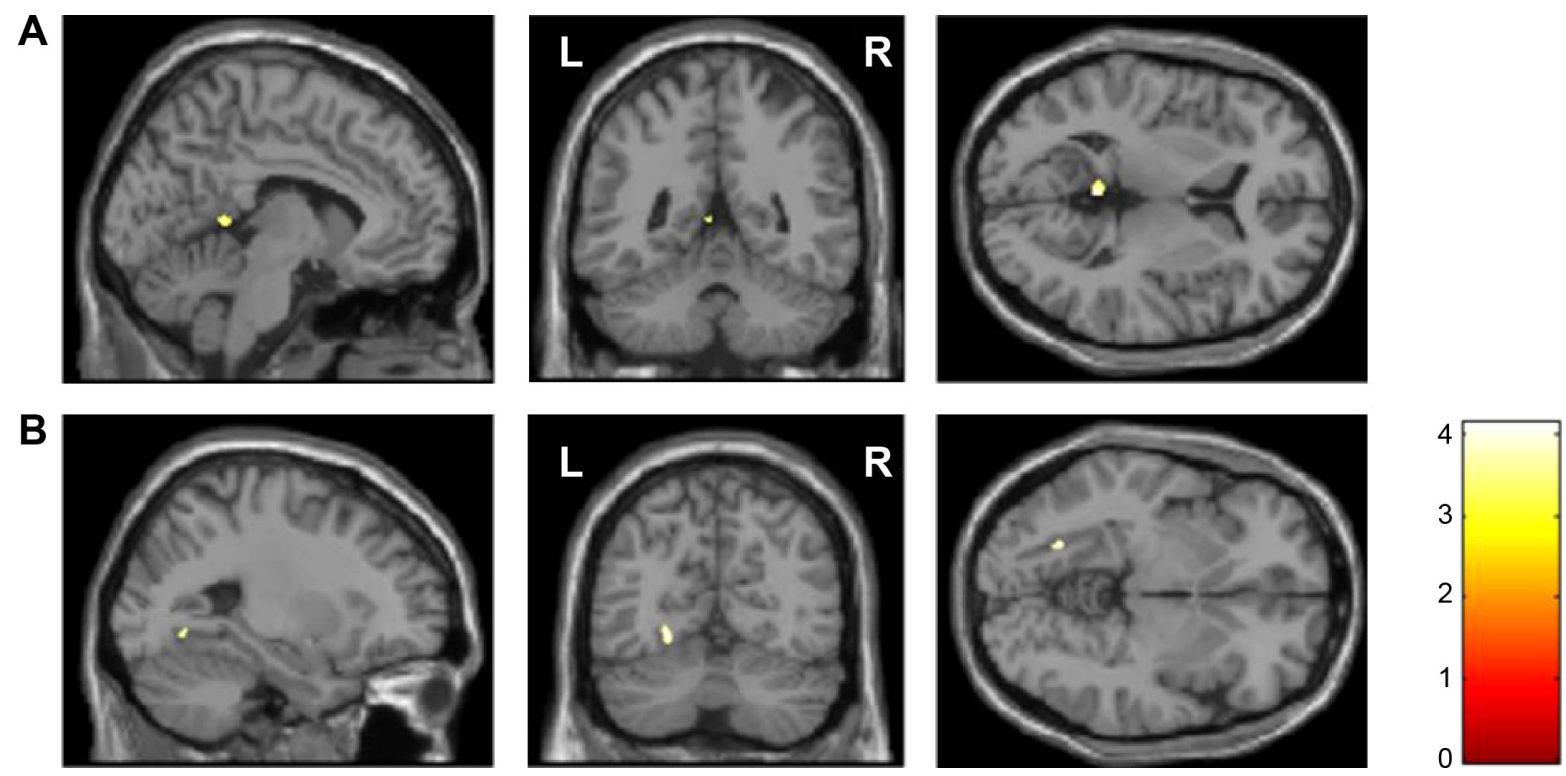

Figure I Regions where there are gray-matter volume abnormalities between unmedicated major depressive disorder patients and healthy controls.

Notes: Significant group differences were identified by $t$-test $(P<0.001$, uncorrected, cluster size greater than 150 voxels). The color bar represents the $t$-values of two sample $t$-test. Regions with increased gray-matter volume in patients were located in (A) the left posterior cingulate gyrus; regions with decreased gray-matter volume in patients were located in (B) the left lingual gyrus.

Abbreviations: L, left; R, right.

Besides, the LG was reported to be involved in the visual recognition network and play a key role in global aspects of figure recognition ${ }^{56}$ and object naming. ${ }^{57}$ In addition, it was believed to be involved in the perception of emotions when facial stimuli were presented. Much evidence has shown that anatomical changes in the PCG and LG may be associated with dysfunction of emotional processing ${ }^{53,58,59}$ and play a role in emotional processing related to episodic memory. ${ }^{60}$
Alterations in ReHo reflect functional brain spontaneous neuronal activity, and previous findings of ReHo have indicated that alterations in regional spontaneous activity existed in depression subjects, especially in MDD. ${ }^{61}$ On one hand, increased ReHo values in the precuneus has been reported in early onset treatment-naïve depressions. ${ }^{62}$ The authors suggested that the average ReHo values in this region could serve as biomarkers to distinguish patients with early onset
A

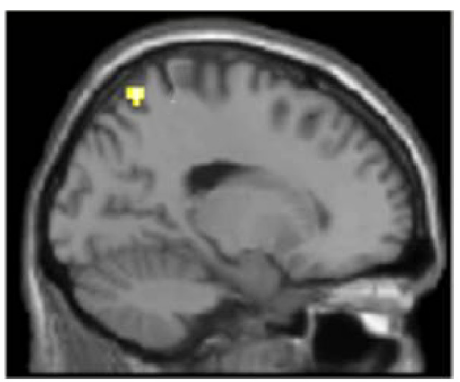

B

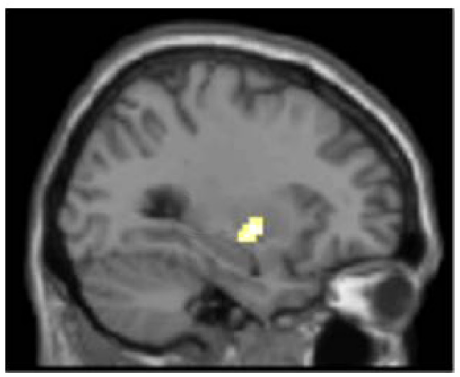

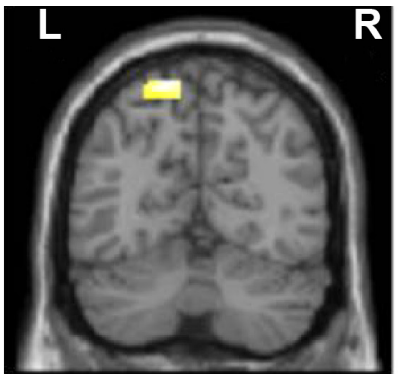

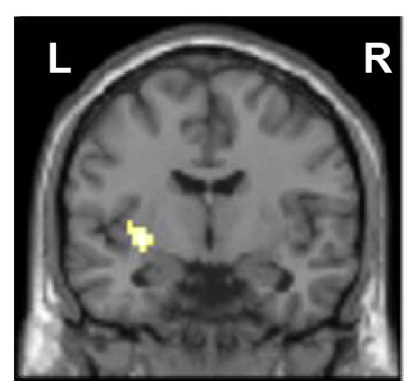

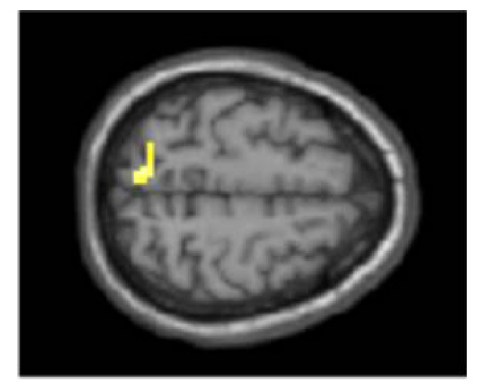

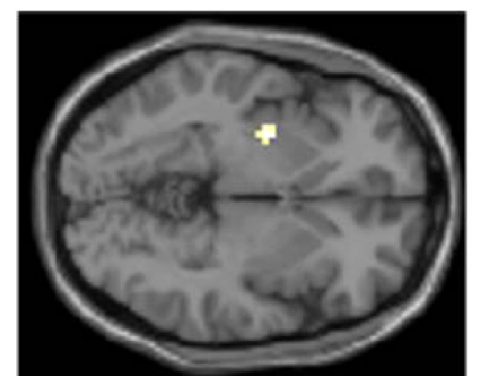

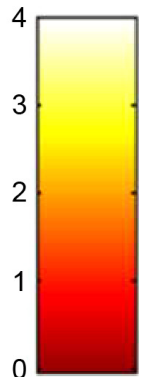

Figure 2 Regional homogeneity abnormalities during resting-state functional imaging between unmedicated major depressive disorder patients and healthy controls.

Notes: Significant group differences were identified by $t$-test $(P<0.00$ I, corrected for multiple comparisons with the AlphaSim program). The color bar represents the $t$-values of two sample $t$-test. Regions showing increased regional homogeneity in patients were located in $(\mathbf{A})$ the left precuneus; regions with decreased regional homogeneity in patients were located in (B) the left putamen.

Abbreviations: L, left; $R$, right. 
Table 2 GMV and ReHo comparison between 50 MDD patients and $50 \mathrm{HCs}$

\begin{tabular}{|c|c|c|c|c|c|c|}
\hline Regions & MNI coordinates $(x, y, z)^{a}$ & Cluster size & $P$-value ${ }^{b}$ & $t^{c}$ & L/R & BA \\
\hline \multicolumn{7}{|l|}{ GMV } \\
\hline \multicolumn{7}{|l|}{$\mathrm{MDD}>\mathrm{HCs}$} \\
\hline Posterior cingulate gyrus & $-3,-48,8$ & 208 & 0.001 & 4.1522 & L & 29 \\
\hline \multicolumn{7}{|l|}{$\mathrm{MDD}<\mathrm{HCs}$} \\
\hline Lingual gyrus & $-25,-64,-4$ & 251 & 0.001 & 3.9195 & L & 19 \\
\hline \multicolumn{7}{|l|}{ ReHo } \\
\hline \multicolumn{7}{|l|}{$\mathrm{MDD}>\mathrm{HCs}$} \\
\hline Precuneus & $-12,-63,60$ & 44 & 0.001 & 4.4111 & L & 7 \\
\hline \multicolumn{7}{|l|}{$\mathrm{MDD}<\mathrm{HCs}$} \\
\hline Putamen & $-27,-6,-3$ & 28 & 0.001 & 3.9822 & L & - \\
\hline
\end{tabular}

Notes: aCoordinates of primary peak locations in the MNI space; buncorrected; 'peak voxel showing gray-matter difference among the MDD and HC groups.

Abbreviations: GMV, gray-matter volume; ReHo, regional homogeneity; MDD, major depressive disorder; HCs, healthy controls; MNI, Montreal Neurological Institute; L, left; R, right; BA, Brodmann area.

depression from individuals with late-onset depression. In the current study, ReHo values of depression patients were found to be increased in the posterior components of the default mode network (precuneus), which was implicated in broad-based continuous sampling of external and internal environments and appeared to serve an important adaptive function. In addition, the alteration in brain regional activity in this region may be implicated in working memory dysfunction ${ }^{63}$ and may be associated with the cognitive processing of depression. ${ }^{64,65}$ On the other hand, decreased brain activity in the putamen is consistent with a previous study. ${ }^{66}$ A study further reported that decreased ReHo value in the putamen plays a key role in planning and execution of a self-generated novel action. ${ }^{67}$ All these findings suggest that abnormalities in the precuneus and putamen may be associated with execution function and play a crucial role in the pathogenesis of depression.

As mentioned earlier, brain structural and functional changes may play a different role in depression. Our current study found that anatomical and resting-state functional deficits existed in different brain regions as a dissociation pattern of brain structural and functional deficits in MDD, especially in GMV and ReHo analysis. Some researchers have found a dissociation pattern of morphometric and functional abnormalities

Table 3 The relationship between GMV and HAM-D or HAM-A

\begin{tabular}{|c|c|c|c|c|}
\hline & \multicolumn{2}{|c|}{ HAM-D } & \multicolumn{2}{|c|}{ HAM-A } \\
\hline & $r$ & $P$-value & $r$ & $P$-value \\
\hline \multicolumn{5}{|l|}{ GMV } \\
\hline Posterior cingulate gyrus & 0.223 & 0.128 & -0.003 & 0.986 \\
\hline Occipital lingual gyrus & 0.192 & 0.190 & 0.216 & 0.140 \\
\hline \multicolumn{5}{|l|}{ ReHo } \\
\hline Precuneus & -0.250 & 0.087 & -0.216 & 0.141 \\
\hline Putamen & 0.034 & 0.818 & 0.203 & 0.166 \\
\hline
\end{tabular}

Abbreviations: GMV, gray-matter volume; ReHo, regional homogeneity; HAM-D, Hamilton Depression Rating Scale; HAM-A, Hamilton Anxiety Rating Scale. in psychiatric disorders, such as schizophrenia ${ }^{30,68}$ and depression..$^{33}$ To the best of our knowledge, this is the first study to use VBM-DARTEL and ReHo methods to reveal both anatomical and functional alterations simultaneously in the same unmedicated patients with MDD. These findings have generated some possible explanations. As a previous study speculated, the dissociation pattern may have resulted from different analysis methods. ${ }^{33}$ Structure MRI is used to identify anatomical deficits in gray matter, while fMRI is used to investigate resting-state functional alterations in gray matter. On the other hand, anatomical and functional abnormalities might play different roles in the disease and contribute independently to the neurobiology of MDD. Anatomical alterations evolve in a relatively static or slow process, ${ }^{68}$ which may represent more stable and long-standing changes. ${ }^{69,70}$ Nevertheless, functional deficits may represent physiological changes related to the acute-illness stage, ${ }^{71}$ and may be normalized after treatment or clinical remission. ${ }^{72,73}$ Furthermore, some studies have reported that anatomical alterations in brain regions may correlate with rumination ${ }^{74}$ or suicide attempts in depression patients, ${ }^{75}$ while functional alteration of the brain was found to be possibly related to refractoriness to treatment. The latter change might be an imaging marker for predicting future depression recurrence. ${ }^{76}$

From the current study, we tentatively speculate that structural and functional deficits are distributed in different brain regions in the same sample of MDD patients, thus providing evidence for the dissociation of the GMV and ReHo changes in depression. The strength of our study was the inclusion of medication-free patients, excluding the possibility of medication. Still, our study had four limitations. First, the small sample size may have reduced the general definitiveness of our results, and well-designed studies with a larger sample size are needed. Second, we did not obtain life-duration data of 
the patients. It is therefore difficult to conclude an association between brain alterations and disease duration. Third, neuropsychological tests were not performed in our study. Fourth, although a dissociation pattern of brain regions with GMV and ReHo alterations was observed in unmedicated patients with MDD, the potential roles of functional and structural changes and the interaction between them need further exploration. Finally, a follow-up study is needed to clarify the relationship between depression and those significant brain alterations.

\section{Conclusion}

Our study suggests a dissociation pattern of brain regions with anatomical and functional alterations in unmedicated patients with MDD, especially with regard to GMV and ReHo. This finding implies that functional and anatomical abnormalities of brain regions might contribute independently to the pathophysiology of MDD.

\section{Acknowledgments}

This research was partly funded by State 863 Projects (2015AA020513) and the National Nature Science Foundation of China (91232711).

\section{Author contributions}

All authors contributed toward data analysis, drafting, and revising the paper, and agree to be accountable for all aspects of the work.

\section{Disclosure}

The authors report no conflicts of interest in this work.

\section{References}

1. Murray CJ, Lopez AD. Evidence-based health policy - lessons from the Global Burden of Disease Study. Science. 1996;274(5288):740-743.

2. Kessler RC, Berglund P, Demler O, et al. The epidemiology of major depressive disorder: results from the National Comorbidity Survey Replication (NCS-R). JAMA. 2003;289(23):3095-3105.

3. Fagiolini A, Kupfer DJ. Is treatment-resistant depression a unique subtype of depression? Biol Psychiatry. 2003;53(8):640-648.

4. Drevets WC, Price JL, Furey ML. Brain structural and functional abnormalities in mood disorders: implications for neurocircuitry models of depression. Brain Struct Funct. 2008;213(1-2):93-118.

5. Peng J, Liu J, Nie B, et al. Cerebral and cerebellar gray matter reduction in first-episode patients with major depressive disorder: a voxel-based morphometry study. Eur J Radiol. 2011;80(2):395-399.

6. Husain MM, McDonald WM, Doraiswamy PM, et al. A magnetic resonance imaging study of putamen nuclei in major depression. Psychiatry Res. 1991;40(2):95-99.

7. Krishnan KR, McDonald WM, Doraiswamy PM, et al. Neuroanatomical substrates of depression in the elderly. Eur Arch Psychiatry Clin Neurosci. 1993;243(1):41-46.

8. Fales CL, Barch DM, Rundle MM, et al. Altered emotional interference processing in affective and cognitive-control brain circuitry in major depression. Biol Psychiatry. 2008;63(4):377-384.
9. Vassilopoulou K, Papathanasiou M, Michopoulos I, et al. A magnetic resonance imaging study of hippocampal, amygdala and subgenual prefrontal cortex volumes in major depression subtypes: melancholic versus psychotic depression. J Affect Disord. 2013;146(2):197-204.

10. Videbech P, Ravnkilde B. Hippocampal volume and depression: a metaanalysis of MRI studies. Am J Psychiatry. 2004;161(11):1957-1966.

11. Sheline YI. Neuroimaging studies of mood disorder effects on the brain. Biol Psychiatry. 2003;54(3):338-352.

12. Lai $\mathrm{CH}, \mathrm{Wu}$ YT. Frontal-insula gray matter deficits in first-episode medication-naive patients with major depressive disorder. J Affect Disord. 2014;160:74-79.

13. Son JH, Han DH, Min KJ, Kee BS. Correlation between gray matter volume in the temporal lobe and depressive symptoms in patients with Alzheimer's disease. Neurosci Lett. 2013;548:15-20.

14. Nakano M, Matsuo K, Nakashima M, et al. Gray matter volume and rapid decision-making in major depressive disorder. Prog Neuropsychopharmacol Biol Psychiatry. 2014;48:51-56.

15. Vasic N, Walter H, Hose A, Wolf RC. Gray matter reduction associated with psychopathology and cognitive dysfunction in unipolar depression: a voxel-based morphometry study. J Affect Disord. 2008;109(1-2): $107-116$.

16. Biswal B, Yetkin FZ, Haughton VM, Hyde JS. Functional connectivity in the motor cortex of resting human brain using echo-planar MRI. Magn Reson Med. 1995;34(4):537-541.

17. Wu $\mathrm{T}$, Long $\mathrm{X}$, Zang $\mathrm{Y}$, et al. Regional homogeneity changes in patients with Parkinson's disease. Hum Brain Mapp. 2009;30(5):1502-1510.

18. Liu H, Liu Z, Liang M, et al. Decreased regional homogeneity in schizophrenia: a resting state functional magnetic resonance imaging study. Neuroreport. 2006;17(1):19-22.

19. Cao Q, Zang Y, Sun L, et al. Abnormal neural activity in children with attention deficit hyperactivity disorder: a resting-state functional magnetic resonance imaging study. Neuroreport. 2006;17(10):1033-1036.

20. Shukla DK, Keehn B, Müller RA. Regional homogeneity of fMRI time series in autism spectrum disorders. Neurosci Lett. 2010;476(1):46-51.

21. Qiu C, Liao W, Ding J, et al. Regional homogeneity changes in social anxiety disorder: a resting-state fMRI study. Psychiatry Res. 2011;194(1): $47-53$

22. Liu Z, Xu C, Xu Y, et al. Decreased regional homogeneity in insula and cerebellum: a resting-state fMRI study in patients with major depression and subjects at high risk for major depression. Psychiatry Res. 2010; 182(3):211-215.

23. Drevets WC. Functional anatomical abnormalities in limbic and prefrontal cortical structures in major depression. Prog Brain Res. 2000;126: 413-431.

24. Iwabuchi SJ, Krishnadas R, Li C, Auer DP, Radua J, Palaniyappan L. Localized connectivity in depression: a meta-analysis of resting state functional imaging studies. Neurosci Biobehav Rev. 2015;51:77-86.

25. Yao Z, Wang L, Lu Q, Liu H, Teng G. Regional homogeneity in depression and its relationship with separate depressive symptom clusters: a resting-state fMRI study. J Affect Disord. 2009;115(3):430-438.

26. Wu QZ, Li DM, Kuang WH, et al. Abnormal regional spontaneous neural activity in treatment-refractory depression revealed by resting-state fMRI. Hum Brain Mapp. 2011;32(8):1290-1299.

27. Wang L, Li K, Zhang Q, et al. Short-term effects of escitalopram on regional brain function in first-episode drug-naive patients with major depressive disorder assessed by resting-state functional magnetic resonance imaging. Psychol Med. 2014;44(7):1417-1426.

28. Guo W, Liu F, Xiao C, et al. Dissociation of anatomical and functional alterations of the default-mode network in first-episode, drug-naive schizophrenia. Clin Neurophysiol. Epub 2015 Feb 16.

29. Ren W, Lui S, Deng W, et al. Anatomical and functional brain abnormalities in drug-naive first-episode schizophrenia. Am J Psychiatry. 2013; 170(11):1308-1316.

30. Lui S, Deng W, Huang X, et al. Association of cerebral deficits with clinical symptoms in antipsychotic-naive first-episode schizophrenia: an optimized voxel-based morphometry and resting state functional connectivity study. Am J Psychiatry. 2009;166(2):196-205. 
31. Rubinov M, Bassett DS. Emerging evidence of connectomic abnormalities in schizophrenia. J Neurosci. 2011;31(17):6263-6265.

32. Michael AM, Baum SA, White T, et al. Does function follow form? Methods to fuse structural and functional brain images show decreased linkage in schizophrenia. Neuroimage. 2010;49(3):2626-2637.

33. Guo W, Liu F, Yu M, et al. Functional and anatomical brain deficits in drug-naive major depressive disorder. Prog Neuropsychopharmacol Biol Psychiatry. 2014;54:1-6.

34. First MB. User's Guide for the Structured Clinical Interview for DSM-IV Axis I Disorders SCID-I: Clinician Version. Washington: American Psychiatric Press; 1997.

35. American Psychiatric Association. Diagnostic and Statistical Manual of Mental Disorders. 4th ed. Text revision. Arlington (VA): APA; 2000.

36. Ashburner J. A fast diffeomorphic image registration algorithm. Neuroimage. 2007;38(1):95-113.

37. Chao-Gan Y, Yu-Feng Z. DPARSF: a MATLAB toolbox for "pipeline" data analysis of resting-state fMRI. Front Syst Neurosci. 2010;4:13.

38. Tononi G, McIntosh AR, Russell DP, Edelman GM. Functional clustering: identifying strongly interactive brain regions in neuroimaging data. Neuroimage. 1998;7(2):133-149.

39. Zang Y, Jiang T, Lu Y, He Y, Tian L. Regional homogeneity approach to fMRI data analysis. Neuroimage. 2004;22(1):394-400.

40. Long XY, Zuo XN, Kiviniemi V, et al. Default mode network as revealed with multiple methods for resting-state functional MRI analysis. J Neurosci Methods. 2008;171(2):349-355.

41. Ballmaier M, Toga AW, Blanton RE, et al. Anterior cingulate, gyrus rectus, and orbitofrontal abnormalities in elderly depressed patients: an MRI-based parcellation of the prefrontal cortex. Am J Psychiatry. 2004;161(1):99-108.

42. Pannekoek JN, van der Werff SJ, van den Bulk BG, et al. Reduced anterior cingulate gray matter volume in treatment-naïve clinically depressed adolescents. Neuroimage Clin. 2014;4:336-342.

43. Bijanki KR, Hodis B, Brumm MC, Harlynn EL, McCormick LM. Hippocampal and left subcallosal anterior cingulate atrophy in psychotic depression. PloS One. 2014;9(10):e110770.

44. Ruegg RG, Zisook S, Swerdlow NR. Depression in the aged. An overview. Psychiatr Clin North Am. 1988;11(1):83-99.

45. Mak AK, Wong MM, Han SH, Lee TM. Gray matter reduction associated with emotion regulation in female outpatients with major depressive disorder: a voxel-based morphometry study. Prog Neuropsychopharmacol Biol Psychiatry. 2009;33(7):1184-1190.

46. Caetano SC, Kaur S, Brambilla P, et al. Smaller cingulate volumes in unipolar depressed patients. Biol Psychiatry. 2006;59(8):702-706.

47. Rodríguez-Cano E, Sarró S, Monté GC, et al. Evidence for structural and functional abnormality in the subgenual anterior cingulate cortex in major depressive disorder. Psychol Med. 2014;44(15):3263-3273.

48. Zhao YJ, Du MY, Huang XQ, et al. Brain grey matter abnormalities in medication-free patients with major depressive disorder: a meta-analysis. Psychol Med. 2014;44(14):2927-2937.

49. Porrino LJ, Goldman-Rakic PS. Brainstem innervation of prefrontal and anterior cingulate cortex in the rhesus monkey revealed by retrograde transport of HRP. J Comp Neurol. 1982;205(1):63-76.

50. Olivier B. Serotonin: a never-ending story. Eur J Pharmacol. 2015;753: $2-18$.

51. Reiner A. The Triune Brain in Evolution. Role in Paleocerebral Functions. Paul D. MacLean. Plenum, New York, 1990. xxiv, 672 pp., illus. \$75. Science. 1990;250(4978):303-305.

52. Leech R, Sharp DJ. The role of the posterior cingulate cortex in cognition and disease. Brain. 2014;137(Pt 1):12-32.

53. Vogt BA, Vogt L, Laureys S. Cytology and functionally correlated circuits of human posterior cingulate areas. Neuroimage. 2006;29(2):452-466.

54. Wright P, Albarracin D, Brown RD, Li H, He G, Liu Y. Dissociated responses in the amygdala and orbitofrontal cortex to bottom-up and top-down components of emotional evaluation. Neuroimage. 2008;39(2):894-902.

55. Damasio AR, Van Hoesen G. Emotional disturbances associated with focal lesions of the limbic frontal lobe. In: Heilman KM, Satz P, editors. Neuropsychology of Human Emotion. New York: Guilford Press; 1983: $85-110$
56. Fink GR, Halligan PW, Marshall JC, Frith CD, Frackowiak RS, Dolan RJ. Where in the brain does visual attention select the forest and the trees? Nature. 1996;382(6592):626-628.

57. Kiyosawa M, Inoue C, Kawasaki T, et al. Functional neuroanatomy of visual object naming: a PET study. Graefes Arch Clin Exp Ophthalmol. 1996;234(2):110-115.

58. Grieve SM, Korgaonkar MS, Koslow SH, Gordon E, Williams LM. Widespread reductions in gray matter volume in depression. Neuromage Clin. 2013;3:332-339.

59. Veer IM, Beckmann CF, van Tol MJ, et al. Whole brain resting-state analysis reveals decreased functional connectivity in major depression. Front Syst Neurosci. 2010;4:41.

60. Maddock RJ. The retrosplenial cortex and emotion: new insights from functional neuroimaging of the human brain. Trends Neurosci. 1999; 22(7):310-316.

61. Ma Z, Li R, Yu J, He Y, Li J. Alterations in regional homogeneity of spontaneous brain activity in late-life subthreshold depression. PloS One. 2013;8(1):e53148.

62. Chen JD, Liu F, Xun GL, et al. Early and late onset, first-episode, treatment-naive depression: same clinical symptoms, different regional neural activities. $J$ Affect Disord. 2012;143(1-3):56-63.

63. Castellanos FX, Margulies DS, Kelly C, et al. Cingulate-precuneus interactions: a new locus of dysfunction in adult attention-deficit/hyperactivity disorder. Biol Psychiatry. 2008;63(3):332-337.

64. Buckner RL, Andrews-Hanna JR, Schacter DL. The brain's default network: anatomy, function, and relevance to disease. Ann N Y Acad Sci. 2008;1124:1-38.

65. Broyd SJ, Demanuele C, Debener S, Helps SK, James CJ, SonugaBarke EJ. Default-mode brain dysfunction in mental disorders: a systematic review. Neurosci Biobehav Rev. 2009;33(3):279-296.

66. Tha KK, Terae S, Nakagawa S, et al. Impaired integrity of the brain parenchyma in non-geriatric patients with major depressive disorder revealed by diffusion tensor imaging. Psychiatry Res. 2013;212(3): 208-215.

67. Yuan Y, Zhang Z, Bai F, et al. Abnormal neural activity in the patients with remitted geriatric depression: a resting-state functional magnetic resonance imaging study. $J$ Affect Disord. 2008;111(2-3):145-152.

68. Ren W, Lui S, Deng W, et al. Anatomical and functional brain abnormalities in drug-naive first-episode schizophrenia. Am J Psychiatry. 2013; 170(11):1308-1316.

69. Asami T, Bouix S, Whitford TJ, Shenton ME, Salisbury DF, McCarley RW. Longitudinal loss of gray matter volume in patients with first-episode schizophrenia: DARTEL automated analysis and ROI validation. Neuroimage. 2012;59(2):986-996.

70. Chan RC, Di X, McAlonan GM, Gong QY. Brain anatomical abnormalities in high-risk individuals, first-episode, and chronic schizophrenia: an activation likelihood estimation meta-analysis of illness progression. Schizophr Bull. 2011;37(1):177-188.

71. Bora E, Fornito A, Pantelis C, Yücel M. Gray matter abnormalities in major depressive disorder: a meta-analysis of voxel based morphometry studies. $J$ Affect Disord. 2012;138(1-2):9-18.

72. Norbury R, Selvaraj S, Taylor M, Harmer C, Cowen P. Increased neural response to fear in patients recovered from depression: a $3 \mathrm{~T}$ functional magnetic resonance imaging study. Psychol Med. 2010;40(3): 425-432.

73. Wang L, Hermens DF, Hickie IB, Lagopoulos J. A systematic review of resting-state functional-MRI studies in major depression. J Affect Disord. 2012;142(1-3):6-12.

74. Machino A, Kunisato Y, Matsumoto T, et al. Possible involvement of rumination in gray matter abnormalities in persistent symptoms of major depression: an exploratory magnetic resonance imaging voxel-based morphometry study. J Affect Disord. 2014;168:229-235.

75. Peng $\mathrm{H}, \mathrm{Wu} \mathrm{K}, \mathrm{Li} \mathrm{J}$, et al. Increased suicide attempts in young depressed patients with abnormal temporal-parietal-limbic gray matter volume. J Affect Disord. 2014;165:69-73.

76. Liu C, Ma X, Song L, et al. Alteration of spontaneous neuronal activity within the salience network in partially remitted depression. Brain Res. 2015;1599:93-102. 


\section{Publish your work in this journal}

Neuropsychiatric Disease and Treatment is an international, peerreviewed journal of clinical therapeutics and pharmacology focusing on concise rapid reporting of clinical or pre-clinical studies on a range of neuropsychiatric and neurological disorders. This journal is indexed on PubMed Central, the 'PsycINFO' database and CAS, and is the official journal of The International Neuropsychiatric Association (INA). The manuscript management system is completely online and includes a very quick and fair peer-review system, which is all easy to use. Visit http://www.dovepress.com/testimonials.php to read real quotes from published authors.

\footnotetext{
Submit your manuscript here: http://www.dovepress.com/neuropsychiatric-disease-and-treatment-journal
} 